\title{
A Resistive Mesh Phantom for Assessing the Performance of EIT Systems
}

\author{
Hervé Gagnon*, Martin Cousineau, Andy Adler, and Alzbeta E. Hartinger, Student Member, IEEE
}

\begin{abstract}
Assessing the performance of electrical impedance tomography (EIT) systems usually requires a phantom for validation, calibration or comparison purposes. This paper describes a resistive mesh phantom to assess the performance of EIT systems while taking into account cabling stray effects similar to in vivo conditions. This phantom is built with 340 precision resistors on a printed circuit board (PCB) representing a 2D circular homogeneous medium. It also integrates equivalent electrical models of the $\mathrm{Ag} / \mathrm{AgCl}$ electrode impedances. The parameters of the electrode models were fitted from impedance curves measured with an impedance analyzer. The technique used to build the phantom is general and applicable to phantoms of arbitrary shape and conductivity distribution. We describe three performance indicators that can be measured with our phantom for every measurement of an EIT data frame: signal-to-noise ratio, accuracy, and modeling accuracy. These performance indicators were evaluated on our EIT system under different frame rates and applied current intensities. The performance indicators are dependent on frame rate, operating frequency, applied current intensity, measurement strategy, and inter-modulation distortion when performing simultaneous measurements at several frequencies. These parameter values should therefore always be specified when reporting performance indicators to better appreciate their significance.
\end{abstract}

Index Terms-Electrical impedance tomography, biomedical instrumentation, resistive mesh phantom.

\section{INTRODUCTION}

$\mathbf{E}$ LECTRICAL impedance tomography (EIT) is a biomedical technique for imaging the electrical conductivity distribution of a body section. An EIT system uses body surface electrodes to measure voltages produced while applying low amplitude sinusoidal currents. These voltage measurements are then converted to conductivity images by solving Maxwell's equations using a numerical model combined with an optimization method. EIT can be useful for studying physiological processes that involve flow or changes in volume of air or blood whose conductivity is significantly different from that of other biological tissues. Although EIT images have low spatial resolution compared to other imaging modalities, they can be obtained in real time at high frame rates and the

Copyright (c) 2010 IEEE. Personal use of this material is permitted. However, permission to use this material for any other purposes must be obtained from the IEEE by sending an email to pubs-permissions@ieee.org.

This work was supported in part by the Natural Sciences and Engineering Research Council of Canada, the Canadian Institutes of Health Research, and the Fonds québécois de la recherche sur la nature et les technologies. Asterisk indicates corresponding author.

H. Gagnon*, M. Cousineau, and A. E. Hartinger are with the Institut de génie biomédical, École Polytechnique de Montréal, Montréal, QC H3C 3A7, Canada (e-mail: gagnon@igb.polymtl.ca).

A. Adler is with the Department of Systems and Computer Engineering, Carleton University, Ottawa, ON K1S 5B6, Canada. required instrumentation is small, lightweight, unobtrusive, and inexpensive. The most promising clinical applications for EIT are functional imaging of heart, lung, and brain functions as well as breast cancer screening [1].

Assessing the performance of EIT systems is often required for validation, calibration, and comparison purposes. EIT systems suitable for in vivo imaging are complex systems requiring several closely interacting hardware and software parts. Modifications made to any part of the system have to be experimentally validated in order to confirm any expected benefit to the performance of the whole system. Calibration has to be performed periodically to account for components whose performance varies over time and to ensure the system is accurate whenever it is used. Objective comparison of EIT data from multiple centers requires a standard calibration approach for the EIT equipment used at each center. In order to fully appreciate descriptions of EIT hardware performance in the literature, it would be useful to have clearly defined objective criteria for comparison purposes. Since no standard procedure for testing EIT systems has been defined, performance results are often published using different methodologies or, worse, without any description of the methodology. Because it is difficult to objectively assess the performance of EIT systems in vivo, phantoms are usually preferred.

Two types of phantoms are described in the literature: physical and mesh phantoms [2]. The former consist of a liquid or solid conductive medium that can be imaged by an EIT system using surface electrodes. The conductive medium usually consists of a conductive gel or a saline solution inside which are inserted targets whose conductivity contrasts with that of the medium. Mesh phantoms are composed of impedance elements interconnected in a particular topology. Resistors [3]-[5], combinations of capacitors and resistors [6], [7] as well as active electronic components [7], [8] have been used as impedance elements. Four topologies have been described in the literature: the Cardiff phantom [3], the wheel phantom [6], and two Göttingen phantoms [4], [5]. The Cardiff phantom is composed of 624 resistors which are assembled as shown in Fig. 1(a). The wheel phantom, shown in Fig. 1(b), is composed of 32 impedance elements whose values are unspecified. Fig. 1(c) and (d) show the two Göttingen phantoms which are respectively composed of 65 and 19 resistors. While physical phantoms generate more realistic signals, mesh phantoms provide predictable, stable, and reproducible signals. Mesh phantoms are therefore better suited for objectively assessing the performance of EIT systems in a reproducible manner.

Our objective was to design a phantom with the follow- 


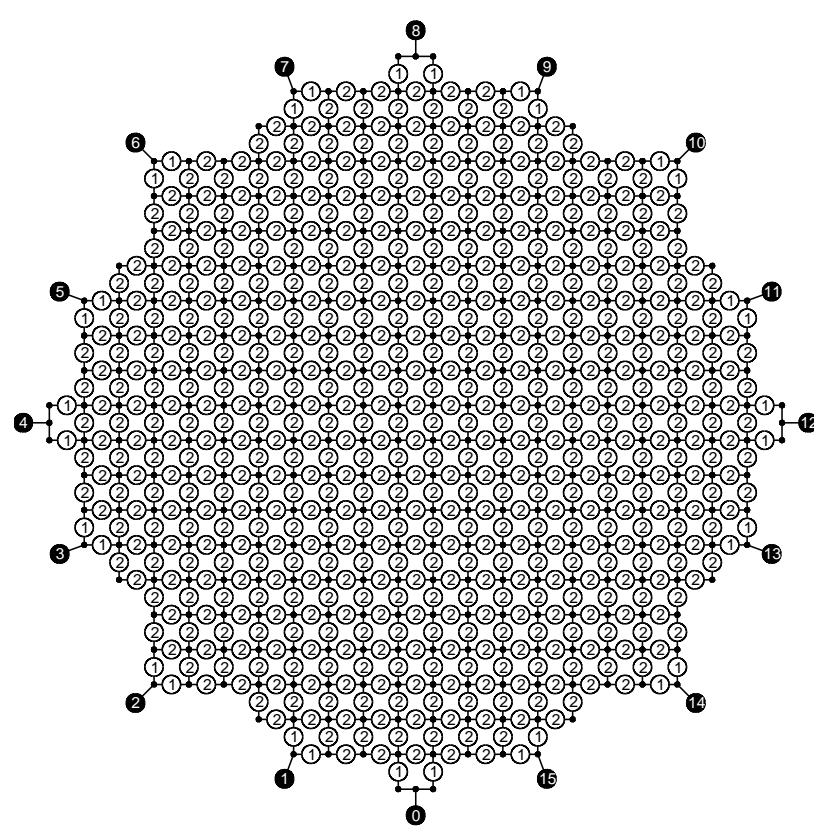

(a)

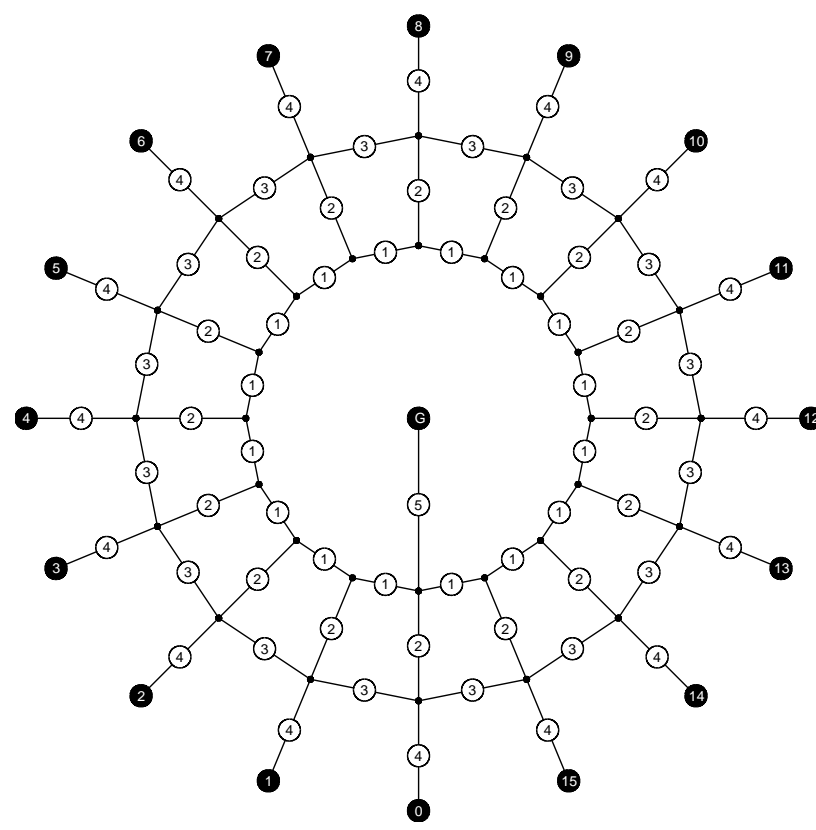

(c)

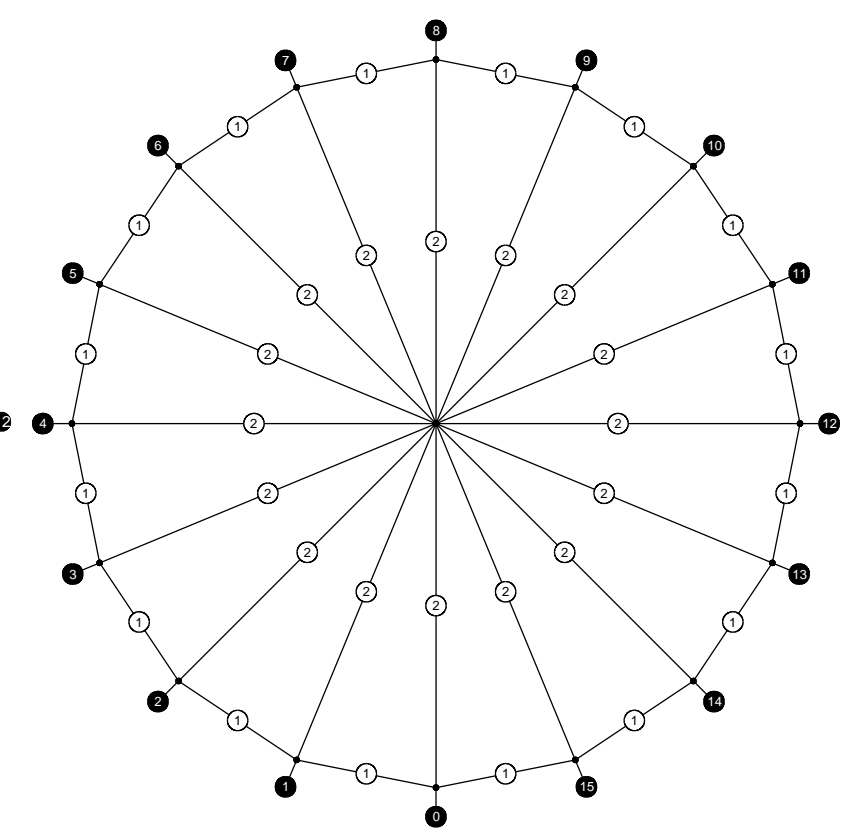

(b)

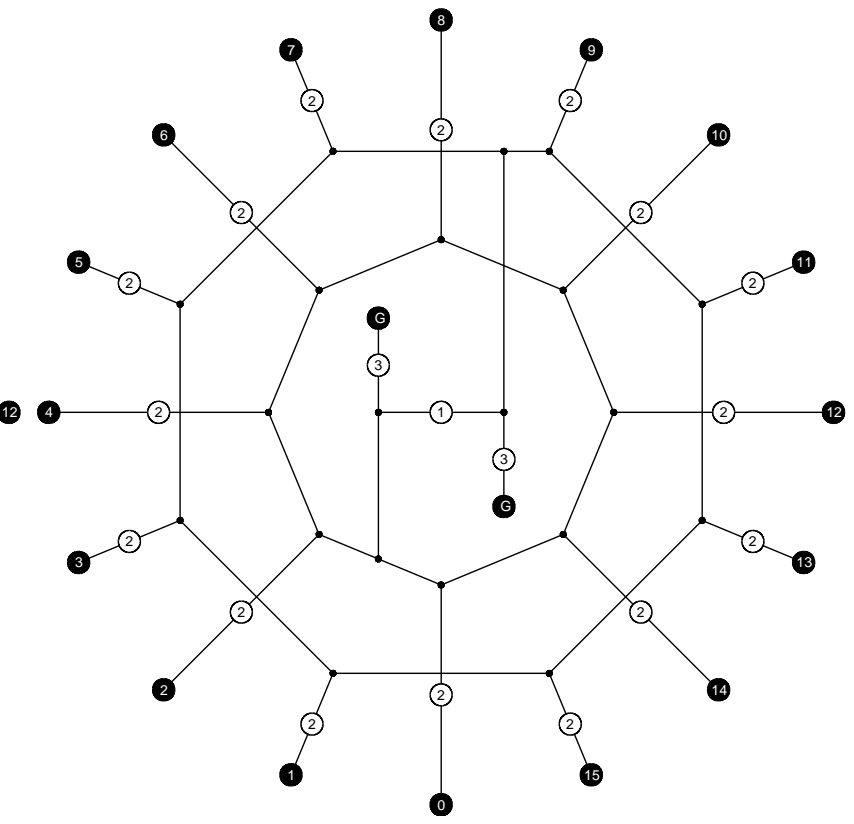

(d)

Fig. 1. (a) Cardiff phantom [3] composed of $51 \Omega$ (1) and $100 \Omega$ (2) resistors. (b) Wheel phantom [6] composed of two different unspecified resistor values identified by white circles. (c) First Göttingen phantom [4] composed of $5 \Omega(1), 60 \Omega(2), 90 \Omega$ (3), $100 \Omega$ (4), and $2.2 \mathrm{k} \Omega$ (5) resistors. (d) Second Göttingen phantom [5] composed of $1 \Omega(1), 121 \Omega$ (2), and $270 \Omega$ (3) resistors. Black circles numbered from 0 to 15 represent the 16 electrode connection sites while black circles identified with the letter $\mathrm{G}$ are associated with ground connection sites.

ing characteristics: 1) approximates a 2D circular continuous homogeneous medium, 2) provides signals with realistic frequency content and amplitude dynamic range, 3) includes a realistic equivalent electrical model of the electrodes, 4) takes into account stray effects similar to in vivo conditions, 5) provides predictable, stable, and reproducible signals, 6) is compatible with all EIT systems, and 7) is capable of producing localized conductivity perturbations. None of the four phantoms described above are able to achieve all these characteristics. As will be shown in section III, the Cardiff phantom and the second Göttingen phantom produce signals whose frequency content and amplitude dynamic range are unrealistic while the wheel phantom and the first Göttingen phantom do not contain enough resistors, especially in the middle, to produce localized conductivity perturbations that can be used to compare reconstruction algorithms.

This paper presents a method for designing mesh phantoms of arbitrary shape and conductivity distributions. The method has been applied to build a phantom composed of 340 precision resistors on a 192 by $192 \mathrm{~mm}$ PCB. The phantom 


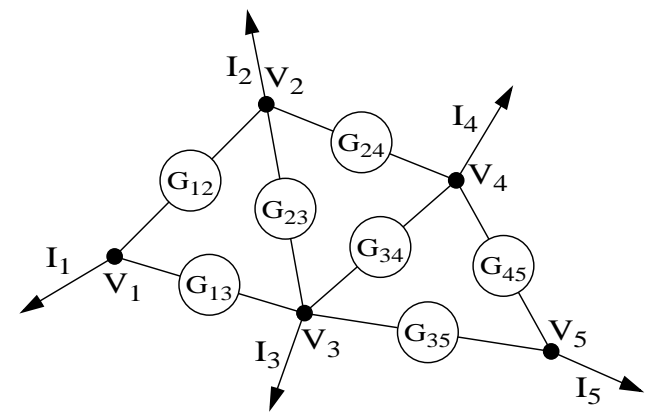

Fig. 2. Sample circuit mesh composed of 5 nodes and 7 admittance elements where $V_{i}$ represents the voltage measured at the $i$ th node, $I_{i}$ represents the outward current at the $i$ th node, and $G_{i j}$ is the admittance value connecting the $i$ th node to the $j$ th node.

can be used to assess the performance of an EIT system by measuring three performance indicators: signal-to-noise ratio, accuracy, and modeling accuracy for every measurement of an EIT data frame. It can also be used to assess the performance of EIT image reconstruction algorithms by producing localized conductivity perturbations. These tests were performed on our EIT system to show how parameters such as frame rate and current intensity affect the three performance indicators.

\section{METHODS}

In order to design phantoms of arbitrary shape and conductivity distribution, we have developed an algorithm based on the analogy between the finite element method (FEM) and a matrix method for solving electrical circuits. The algorithm will be described in the next three sections. The first section shows a matrix method to solve electrical circuits, the second section summarizes the FEM equations, and the third section shows how the two methods are combined to obtain a mesh phantom with the desired shape and conductivity distribution.

\section{A. Matrix method to solve electrical circuits}

The matrix method for solving electrical circuit will be illustrated using the sample circuit mesh shown in Fig. 2. This circuit mesh is composed of 5 nodes and 7 admittance elements where $V_{i}$ represents the voltage measured at the $i$ th node, $I_{i}$ represents the outward current at the $i$ th node, and $G_{i j}$ is the admittance value connecting the $i$ th node to the $j$ th node. By applying Kirchhoff's current law at each circuit node, we obtain the following equations:

$$
\begin{aligned}
& I_{1}=G_{12}\left(V_{2}-V_{1}\right)+G_{13}\left(V_{3}-V_{1}\right) \\
& I_{2}=G_{12}\left(V_{1}-V_{2}\right)+G_{23}\left(V_{3}-V_{2}\right)+G_{24}\left(V_{4}-V_{2}\right) \\
& I_{3}=G_{13}\left(V_{1}-V_{3}\right)+G_{23}\left(V_{2}-V_{3}\right)+G_{34}\left(V_{4}-V_{3}\right) \\
&+G_{35}\left(V_{5}-V_{3}\right) \\
& I_{4}=G_{24}\left(V_{2}-V_{4}\right)+G_{34}\left(V_{3}-V_{4}\right)+G_{45}\left(V_{5}-V_{4}\right) \\
& I_{5}=G_{35}\left(V_{3}-V_{5}\right)+G_{45}\left(V_{4}-V_{5}\right)
\end{aligned}
$$

By converting these equations into matrix form, we obtain $\boldsymbol{Y} \boldsymbol{V}=\boldsymbol{I}$

$$
\begin{aligned}
& \boldsymbol{Y}=\left[\begin{array}{cc}
-\left(G_{12}+G_{13}\right) & G_{12} \\
G_{12} & -\left(G_{12}+G_{23}+G_{24}\right) \\
G_{13} & G_{23} \\
0 & G_{24} \\
0 & 0
\end{array}\right. \\
& G_{23} \\
& -\left(G_{13}+G_{23}+G_{34}+G_{35}\right) \\
& G_{34} \\
& G_{35} \\
& 0 \quad 0 \\
& G_{24} \\
& G_{34} \quad G_{35} \\
& \left.\begin{array}{cc}
-\left(G_{24}+G_{34}+G_{45}\right) & G_{45} \\
G_{45} & -\left(G_{35}+G_{45}\right)
\end{array}\right]
\end{aligned}
$$

and

$$
\begin{aligned}
\boldsymbol{V} & =\left[\begin{array}{lllll}
V_{1} & V_{2} & V_{3} & V_{4} & V_{5}
\end{array}\right]^{T} \\
\boldsymbol{I} & =\left[\begin{array}{lllll}
I_{1} & I_{2} & I_{3} & I_{4} & I_{5}
\end{array}\right]^{T}
\end{aligned}
$$

where $\boldsymbol{A}^{T}$ represents the transpose of matrix $\boldsymbol{A}$.

From this simple example, a general method can be inferred to directly obtain the admittance matrix $\boldsymbol{Y}$ from Fig. 2. Offdiagonal elements $y_{i j}$ and $y_{j i}$ are equal to $G_{i j}$ while diagonal elements $y_{i i}$ are equal to minus the sum of all admittance elements connected to the $i$ th node.

\section{B. FEM formulation}

On a continuous conductive medium, such as those typically used in EIT at low frequencies, Maxwell's equations can be simplified to the following equation:

$$
\nabla \cdot(\sigma \nabla V)=0
$$

where $\sigma$ represents the electrical conductivity and $V$ represents the electrical potential. This equation must be solved over the entire continuous medium $\Omega$. On the boundary $\Gamma$, the following boundary condition applies:

$$
J_{n}=\sigma \nabla V \cdot \boldsymbol{n}
$$

where $\boldsymbol{n}$ represents a unit vector normal to the boundary $\Gamma$ and $J_{n}$ represents the normal current density. Since the two previous equations cannot be solved analytically for a medium of arbitrary shape and conductivity distribution, the FEM will be used. The FEM consists in subdividing the continuous medium into a mesh of triangular elements inside which the conductivity is assumed constant and the electric potential varies linearly. By applying the FEM with linear interpolation on a triangle, the following elementary system of equations can be obtained [9]:

$$
\boldsymbol{Y}_{\mathrm{e}} \boldsymbol{V}_{\mathrm{e}}=\boldsymbol{I}_{\mathrm{e}} \Leftrightarrow\left[\begin{array}{lll}
y_{11} & y_{12} & y_{13} \\
y_{21} & y_{22} & y_{23} \\
y_{31} & y_{32} & y_{33}
\end{array}\right]\left[\begin{array}{c}
V_{1} \\
V_{2} \\
V_{3}
\end{array}\right]=\left[\begin{array}{c}
i_{1} \\
i_{2} \\
i_{3}
\end{array}\right]
$$




$$
\begin{aligned}
y_{i j} & =\oint_{\Omega_{\mathrm{e}}} \sigma_{\mathrm{e}}\left(\frac{\partial f_{i}}{\partial x} \frac{\partial f_{j}}{\partial x}+\frac{\partial f_{i}}{\partial y} \frac{\partial f_{j}}{\partial y}\right) d \Omega_{\mathrm{e}} \\
i_{j} & =\oint_{\Gamma_{\mathrm{e}}} f_{j} J_{n} d \Gamma_{\mathrm{e}}
\end{aligned}
$$

$$
\begin{gathered}
{\left[\begin{array}{ll}
f_{1}(x, y) & f_{2}(x, y) \\
f_{3}(x, y)
\end{array}\right]=} \\
{\left[\begin{array}{lll}
x & y & 1
\end{array}\right]\left[\begin{array}{lll}
x_{1} & y_{1} & 1 \\
x_{2} & y_{2} & 1 \\
x_{3} & y_{3} & 1
\end{array}\right]^{-1}}
\end{gathered}
$$

where $\left(x_{i}, y_{i}\right)$ represents the $i$ th triangle vertex coordinates and $V_{i}$ represents the electrical potential at the $i$ th triangle vertex. $\boldsymbol{Y}_{\mathrm{e}}$ is called the elementary admittance matrix, $\boldsymbol{V}_{\mathrm{e}}$, the elementary potential vector, and $\boldsymbol{I}_{\mathrm{e}}$, the elementary current vector. By developing (14) and computing the $f_{i}$ derivatives, we obtain:

$$
\begin{aligned}
& \frac{\partial f_{1}}{\partial x}=\frac{\left(y_{2}-y_{3}\right)}{\Delta}, \frac{\partial f_{2}}{\partial x}=\frac{\left(y_{3}-y_{1}\right)}{\Delta}, \frac{\partial f_{3}}{\partial x}=\frac{\left(y_{1}-y_{2}\right)}{\Delta} \\
& \frac{\partial f_{1}}{\partial y}=\frac{\left(x_{3}-x_{2}\right)}{\Delta}, \frac{\partial f_{2}}{\partial y}=\frac{\left(x_{1}-x_{3}\right)}{\Delta}, \frac{\partial f_{3}}{\partial y}=\frac{\left(x_{2}-x_{1}\right)}{\Delta}
\end{aligned}
$$

$$
\Delta=x_{1} y_{2}-x_{1} y_{3}-x_{2} y_{1}+x_{2} y_{3}+x_{3} y_{1}-x_{3} y_{2}
$$

By substituting these equations into (12), knowing $\sigma_{\mathrm{e}}$ is constant inside an element and the area of an element is given by $\Delta / 2$, we obtain:

$$
\begin{aligned}
& \boldsymbol{Y}_{\mathrm{e}}=\left[\begin{array}{ccc}
-\left(G_{12}+G_{13}\right) & G_{12} & G_{13} \\
G_{12} & -\left(G_{12}+G_{23}\right) & G_{23} \\
G_{13} & G_{23} & -\left(G_{13}+G_{23}\right)
\end{array}\right] \\
& G_{12}=\frac{\sigma_{\mathrm{e}}}{2 \Delta}\left[\left(y_{2}-y_{3}\right)\left(y_{3}-y_{1}\right)+\left(x_{3}-x_{2}\right)\left(x_{1}-x_{3}\right)\right] \\
& G_{13}=\frac{\sigma_{\mathrm{e}}}{2 \Delta}\left[\left(y_{2}-y_{3}\right)\left(y_{1}-y_{2}\right)+\left(x_{3}-x_{2}\right)\left(x_{2}-x_{1}\right)\right] \\
& G_{23}=\frac{\sigma_{\mathrm{e}}}{2 \Delta}\left[\left(y_{3}-y_{1}\right)\left(y_{1}-y_{2}\right)+\left(x_{1}-x_{3}\right)\left(x_{2}-x_{1}\right)\right]
\end{aligned}
$$

Elementary matrices $\boldsymbol{Y}_{\mathrm{e}}, \boldsymbol{V}_{\mathrm{e}}$, and $\boldsymbol{I}_{\mathrm{e}}$ computed for every triangular element must then be respectively assembled into the global admittance matrix $\boldsymbol{Y}$, the global potential vector $\boldsymbol{V}$, and the global current vector $\boldsymbol{I}$. This is performed by assigning a global node number to each of the $n$ nodes composing the triangular FEM mesh and adding the contribution of each elementary matrix to the corresponding global matrix taking into account the assigned global node number of every triangle vertex. The global matrix dimensions are therefore $(n \times n)$ for $\boldsymbol{Y}$ and $(n \times 1)$ for $\boldsymbol{V}$ and $\boldsymbol{I}$.

\section{Method for building phantoms of arbitrary shape and conductivity distributions}

The method for building phantoms of arbitrary shape and conductivity distributions is based on the similarities between the two methods presented in the previous sections. Both methods provide a $\boldsymbol{Y} \boldsymbol{V}=\boldsymbol{I}$ formulation, the first one for an electrical circuit mesh and the second one for a continuous conductive medium. When comparing (6) and (18), the

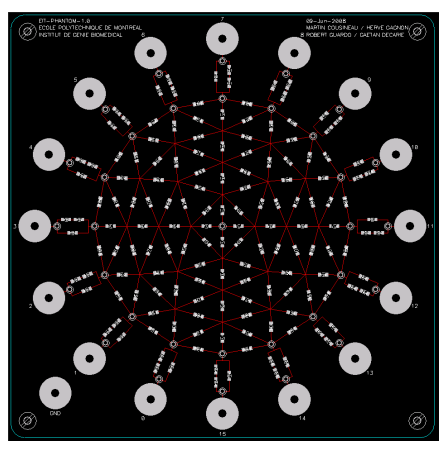

(a)

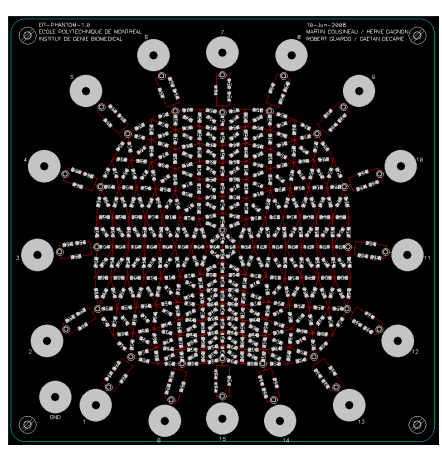

(c)

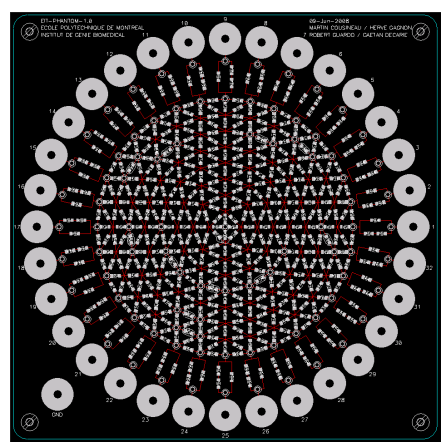

(b)

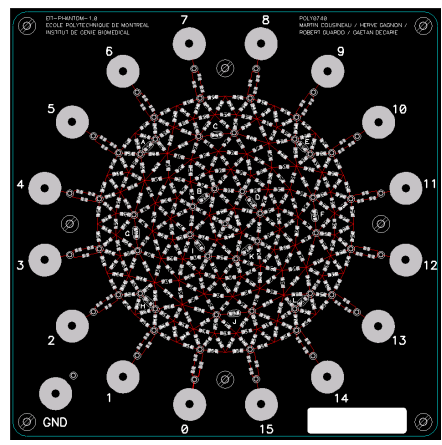

(d)
Fig. 3. (a) Circular phantom with 16 electrodes. (b) Circular phantom with 32 electrodes. (c) Torso-shaped phantom with 16 electrodes. (d) Phantom selected for fabrication.

similarities between the admittance matrices obtained by both methods are obvious.

The method for building phantoms of arbitrary shape and conductivity distribution can be summarized with the following steps: 1) Define the desired shape and conductivity distribution for the phantom. 2) Divide the desired shape into a triangular mesh from which every triangle corresponds to an element of the FEM model and every triangle side corresponds to a resistor of the mesh phantom. 3) Compute the elementary admittance matrix $\boldsymbol{Y}_{\mathrm{e}}$ for every triangle of the mesh. 4) Assemble all elementary admittance matrices into a global admittance matrix $\boldsymbol{Y}$. 5) Extract each resistor value composing the mesh phantom from the global admittance matrix $\boldsymbol{Y}$. The resistor value connecting the $i$ th node of the mesh phantom to the $j$ th node is equal to the inverse of the $(i, j)$ element of $\boldsymbol{Y}$.

By selecting the same triangular mesh topology for the phantom and the FEM model, the designed phantom will theoretically have the same accuracy as the FEM model. In practice, however, this accuracy is slightly reduced by the fact that resistors of standard nominal values with limited tolerance are used to build the phantom.

\section{Phantom designed with the proposed method}

The method described in the previous section has been programmed with the added possibility of automatically generating Gerber files that can be directly used for PCB manufacture. To increase realism, we also added an equivalent electrical model of the electrodes that will be described in the following 


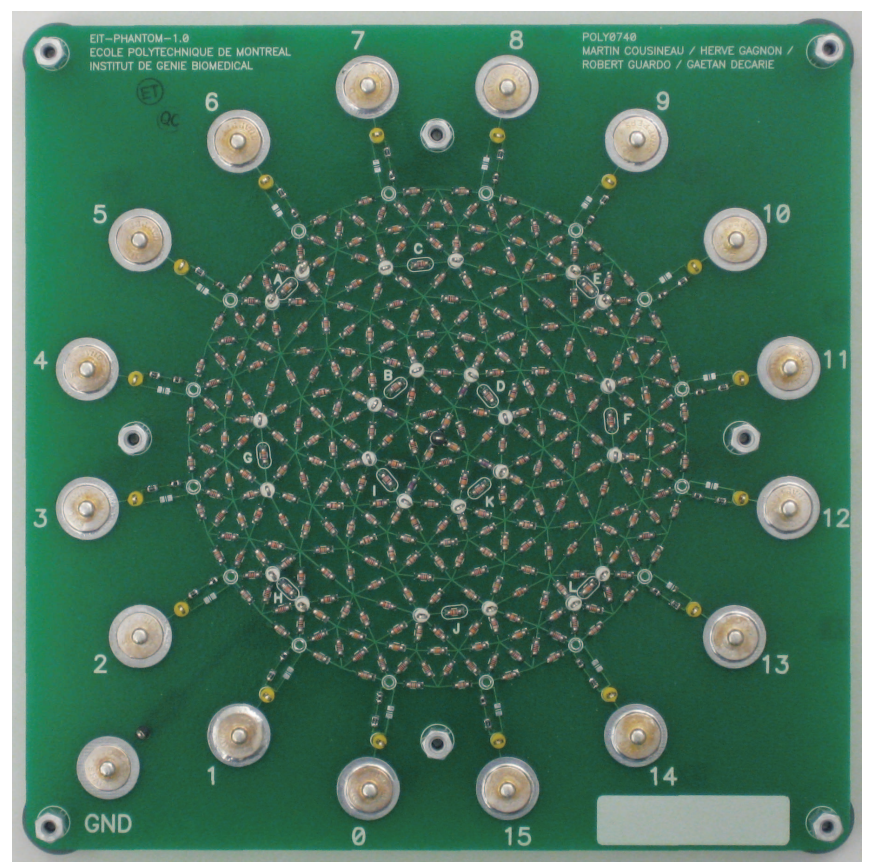

(a)

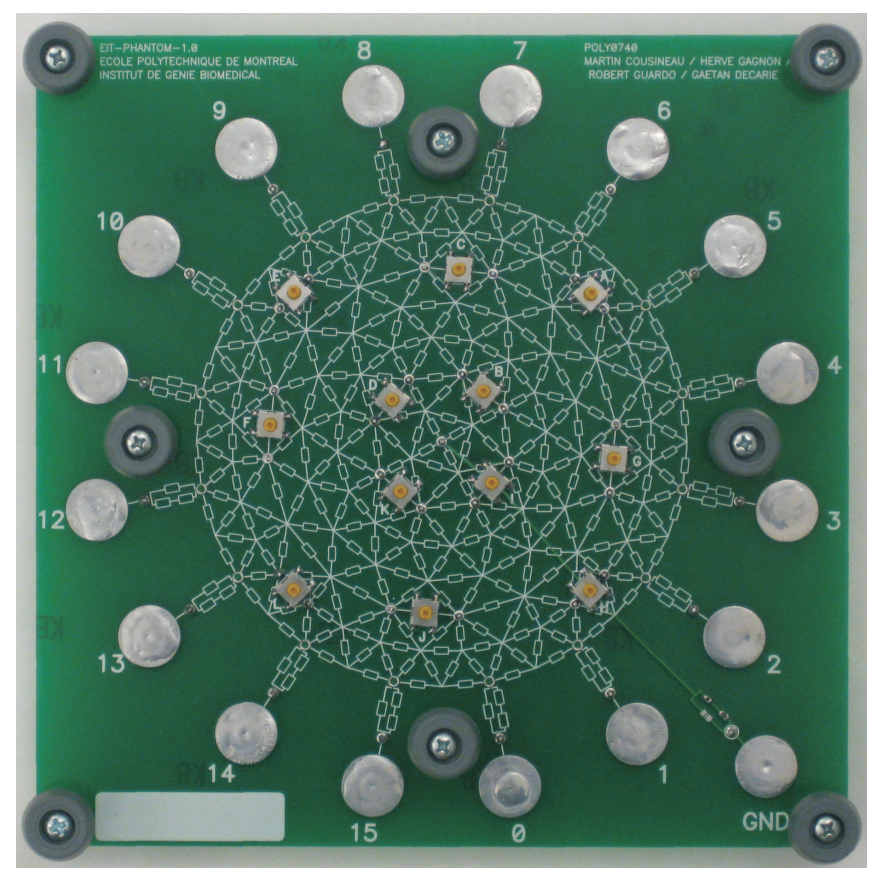

(b)

Fig. 4. (a) Top and (b) bottom views of the resistor mesh phantom.

section. To prove the generality of the method, Fig. 3 shows CAD-software screen snapshots of four different phantoms designed with the proposed method. These phantoms are circular or torso-shaped and have either 16 or 32 electrodes. In order to obtain the characteristics described in the introduction, we have built the phantom shown in Fig. 3(d) representing a 2D circular homogeneous medium. This phantom includes $3400.1 \%$ precision resistors with a temperature coefficient of $25 \mathrm{ppm} / \mathrm{K}, 17$ snap-on connectors (including the ground connection), and 12 switches that can be used to short-circuit

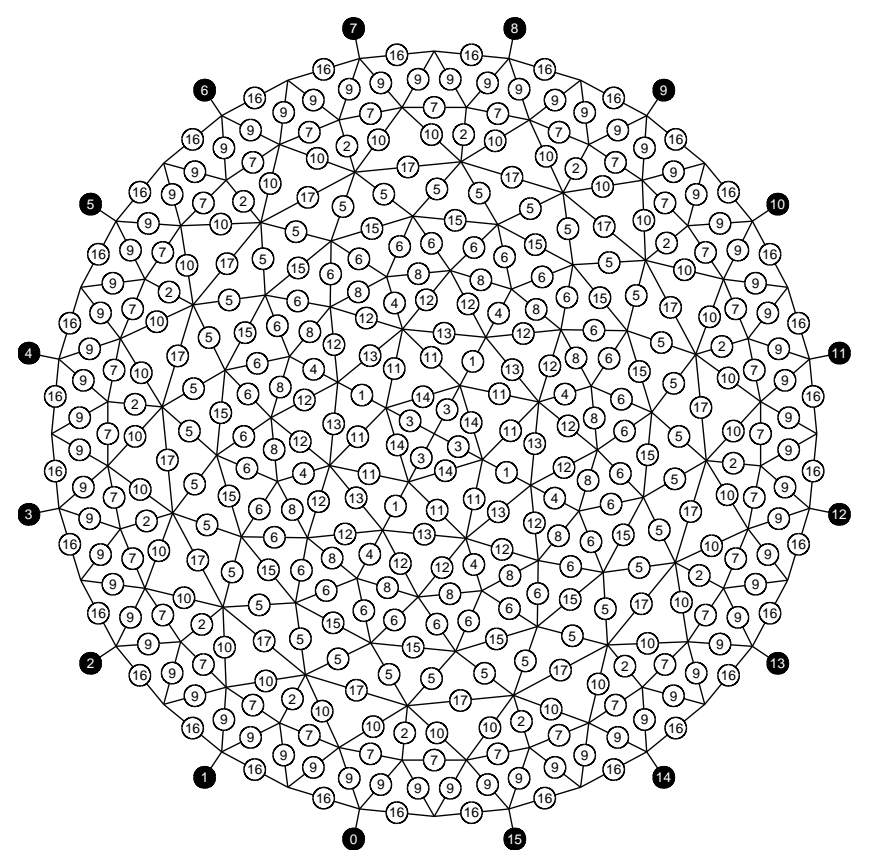

Fig. 5. Schematic representation of the resistor mesh phantom. White circles numbered from 1 to 17 represent resistors whose nominal values are given in Table I. Black circles numbered from 0 to 15 represent the electrode connection sites.

TABLE I

NOMINAL VALUES FOR THE NUMBERED RESISTORS REPRESENTED IN FIG. 5

\begin{tabular}{ccc}
\hline Resistor number & Quantity & Nominal value $(\Omega)$ \\
\hline 1 & 4 & 51.1 \\
2 & 16 & 59.0 \\
3 & 4 & 63.4 \\
4 & 8 & 64.9 \\
5 & 32 & 71.5 \\
6 & 32 & 90.9 \\
7 & 32 & 95.3 \\
8 & 16 & 97.6 \\
9 & 64 & 100 \\
10 & 32 & 130 \\
11 & 8 & 133 \\
12 & 16 & 147 \\
13 & 8 & 178 \\
14 & 4 & 200 \\
15 & 16 & 261 \\
16 & 32 & 365 \\
17 & 16 & 3160 \\
\hline
\end{tabular}

individual resistors to produce localized conductivity perturbations. Top and bottom views of the phantom PCB are shown in Fig. 4. A schematic representation of the resistor mesh topology is shown in Fig. 5 where white circles numbered from 1 to 17 represent resistors whose nominal values are given in Table I. 


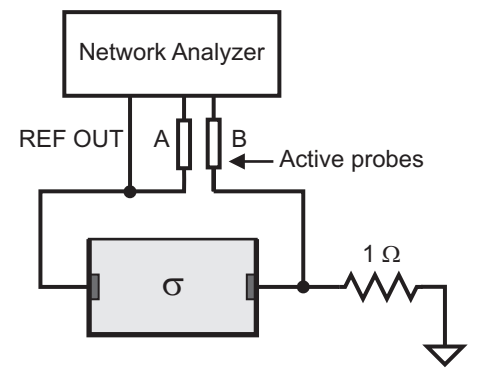

Fig. 6. Electrode impedance measurement set-up.

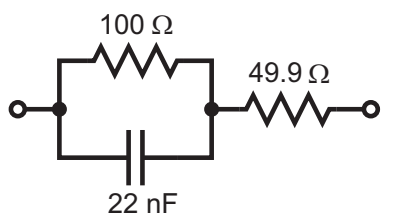

Fig. 7. Equivalent electrical model of an $\mathrm{Ag} / \mathrm{AgCl}$ electrode.

\section{E. Equivalent electrical model of an electrode}

We measured the impedance of two $\mathrm{Ag} / \mathrm{AgCl}$ electrodes immersed in a saline solution from $10 \mathrm{~Hz}$ to $1 \mathrm{MHz}$ using the set-up illustrated in Fig. 6 and a network/spectrum/impedance analyzer (4395A, Agilent Technologies). A voltage signal is applied by the network analyzer to the REF OUT output and voltage measurements are performed at the $\mathrm{A}$ and $\mathrm{B}$ inputs using wideband active probes (41800A, Agilent Technologies). The network analyzer then displays the complex ratio of the $\mathrm{A}$ and $\mathrm{B}$ voltage signals which corresponds for this particular set-up to $Z_{\sigma}$ which can be expressed as:

$$
Z_{\sigma}(j \omega)=2 Z_{\text {electrode }}(j \omega)+Z_{\text {saline }}(j \omega)+1 \Omega,
$$

where $\omega$ is the angular frequency, $Z_{\text {electrode }}$, the $\mathrm{Ag} / \mathrm{AgCl}$ electrode impedance, and $Z_{\text {saline }}$, the impedance of the saline
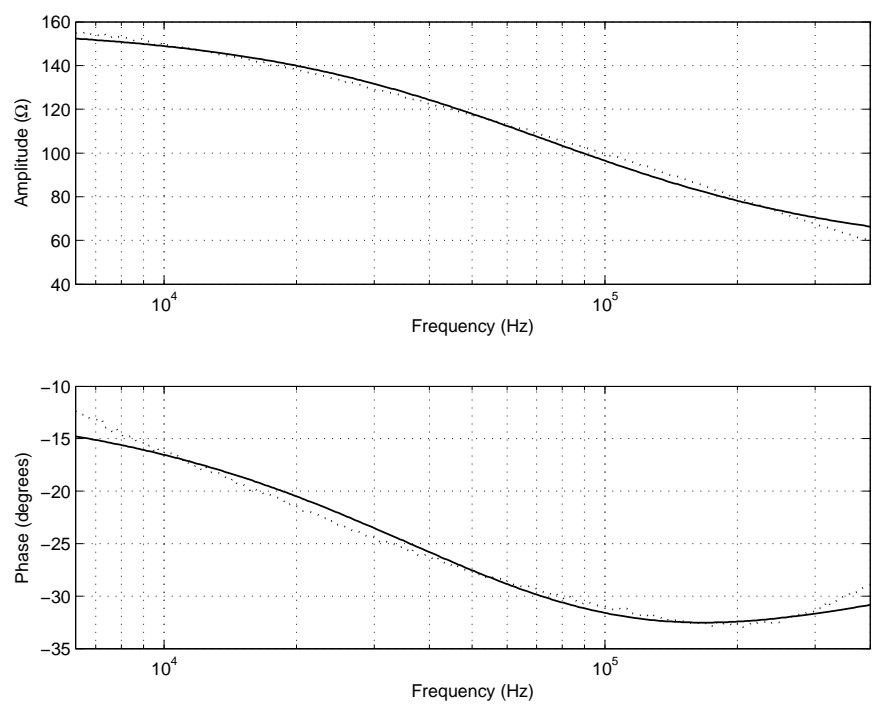

Fig. 8. Electrode impedance spectra obtained from experimental data $(\cdots)$ and from the equivalent electrical model of the electrode whose component values have been optimized by the Levenberg-Marquardt method (-). which is unknown since it depends on the geometry of the saline receptacle. To remove the contribution of the saline solution $\left(Z_{\text {saline }}\right)$ from the measured impedances $Z_{\sigma}$, the measurements were repeated with a saline solution whose conductivity was doubled $\left(Z_{2 \sigma}(j \omega)\right)$. The conductivity of the saline solutions was measured with a conductivity meter (model 1481-40, Cole-Parmer Instrument Company). These measurements can be expressed as:

$$
Z_{2 \sigma}(j \omega)=2 Z_{\text {electrode }}(j \omega)+\frac{1}{2} Z_{\text {saline }}(j \omega)+1 \Omega .
$$

From the two previous equations, we obtain:

$$
Z_{\text {electrode }}(j \omega)=Z_{2 \sigma}(j \omega)-\frac{1}{2} Z_{\sigma}(j \omega)-\frac{1}{2} \Omega \text {. }
$$

To reproduce the complex impedance behavior of the $\mathrm{Ag} / \mathrm{AgCl}$ electrodes, we selected an equivalent electrical model composed of a resistor in series with a parallel combination of a resistor and a capacitor as shown in Fig. 7 [10]. Values for the capacitor and the two resistors were obtained by using the Levenberg-Marquardt [11] method to fit the impedance of the equivalent electrical model to the experimental data measured between 6.25 and $400 \mathrm{kHz}$, which represents six octaves centered at $50 \mathrm{kHz}$, a common operating frequency for EIT systems. Fig. 8 shows the impedance spectra computed from the experimental measurements using (24) and from the equivalent electrical model of an electrode using the resistor and capacitor values obtained from the Levenberg-Marquardt optimization process. These optimized values were rounded to the nearest standard resistor and capacitor values which are specified in Fig. 7. The circuit shown in Fig. 7 was inserted in series with each of the 17 snap-on connectors to reproduce the complex impedance behavior of the $\mathrm{Ag} / \mathrm{AgCl}$ electrodes.

\section{F. Performance indicators}

In order to assess the performance of an EIT system, 1000 data frames, each consisting of $n$ measurements, were acquired on the mesh phantom. The average and variance signals of the 1000 data frames were then computed.

Signal-to-noise ratio (SNR) is computed using the following formula:

$$
\mathrm{SNR}_{i}=20 \log \frac{\left|\mathrm{E}\left[m_{i}\right]\right|}{\sqrt{\operatorname{Var}\left[m_{i}\right]}},
$$

where $m_{i}$ represents the $i$ th measurement, $\mathrm{E}\left[m_{i}\right]$, the average of $m_{i}$, and $\operatorname{Var}\left[m_{i}\right]$, the variance of $m_{i}$.

Accuracy (A) is computed using the following formula:

$$
\mathrm{A}_{i}=\left[1-\left|\frac{\mathrm{E}\left[m_{i}\right]-m_{i}^{\mathrm{T}}}{m_{i}^{\mathrm{T}}}\right|\right] \times 100 \%,
$$

where $m_{i}^{\mathrm{T}}$ represents the theoretical value for the $i$ th measurement.

Some EIT systems have known hardware imperfections that can be accounted for by a calibration procedure performed by the user or a model of hardware imperfections that can be integrated into the forward problem solver of reconstruction algorithms [12]. To account for such systems, modeling accuracy (MA) is defined by the following formula:

$$
\mathrm{MA}_{i}=\left[1-\left|\frac{f_{i}(\boldsymbol{m})-f_{i}^{T}\left(\boldsymbol{m}^{\mathrm{T}}\right)}{f_{i}^{T}\left(\boldsymbol{m}^{\mathrm{T}}\right)}\right|\right] \times 100 \%,
$$



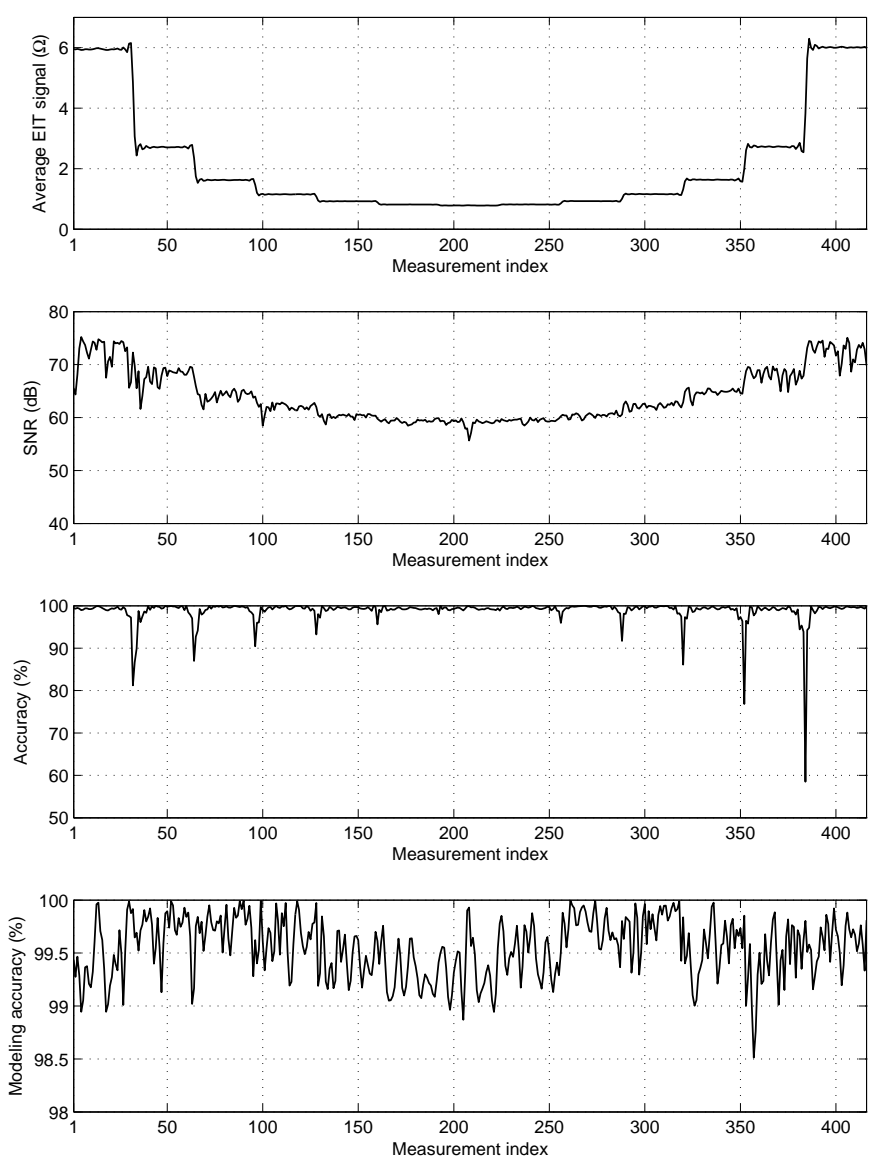

Fig. 9. Performance indicators of our EIT system.

where $\boldsymbol{m}$ represents the $n$-length average measurement vector whose $i$ th element is equal to $\mathrm{E}\left[m_{i}\right], \boldsymbol{m}^{\mathrm{T}}$, the $n$-length theoretical measurement vector whose $i$ th element is equal to $m_{i}^{\mathrm{T}}, f_{i}(\boldsymbol{m})$, the $i$ th measurement corrected by a calibration protocol, and $f_{i}^{T}\left(\boldsymbol{m}^{\mathrm{T}}\right)$, the $i$ th theoretical measurement compensated for any hardware imperfection that can be modeled. Accuracy is therefore evaluated using raw EIT data provided by the EIT system while modeling accuracy is evaluated using data compensated for any known hardware imperfection. For EIT systems that do not implement a calibration procedure and have no model of hardware imperfections that can be integrated into the forward problem solver [12], accuracy and modeling accuracy are equal.

\section{RESULTS}

One thousand measurements were acquired on the phantom with our EIT system [13]. Fig. 9 shows graphically the performance indicators that were obtained at 4.71 frames per second with an applied current intensity of $4 \mathrm{mApp}$ at $50 \mathrm{kHz}$. Table II summarizes the three performance indicators by specifying mean, minimum, and maximum values. The system was set up to acquire data using the measurement sequence illustrated in Fig. 10 which is equivalent to the Sheffield protocol [1] except that measurements are performed in a different sequence. Compared to the Sheffield protocol, this sequence makes it easier to visually pinpoint any flaws
TABLE II

PERFORMANCE INDICATORS OF OUR EIT SYSTEM

\begin{tabular}{lll}
\hline SNR $(\mathrm{dB})$ & mean & 64.03 \\
& maximum & 75.19 \\
& minimum & 55.66 \\
\hline Accuracy $(\%)$ & mean & 98.84 \\
& maximum & 99.99 \\
& minimum & 58.52 \\
\hline Modeling & mean & 99.56 \\
Accuracy (\%) & maximum & 100.0 \\
& minimum & 98.51 \\
\hline
\end{tabular}
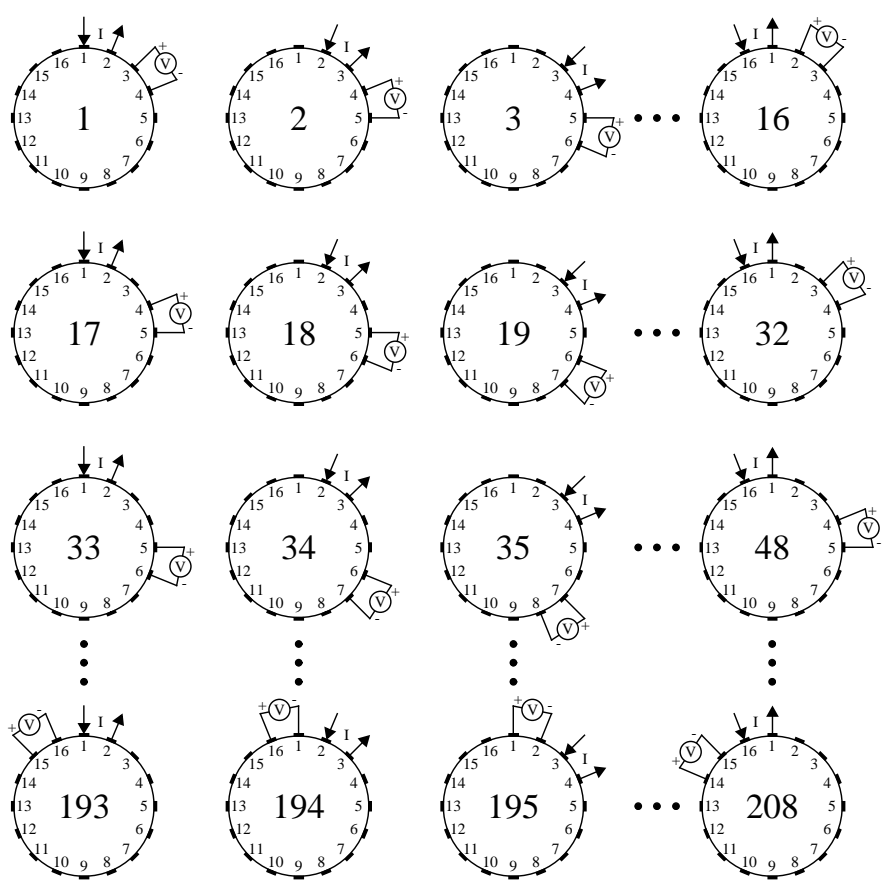

Fig. 10. Measurement sequence used to acquire the EIT data shown in Fig. 9.

in the EIT data because of its regular shape consisting in 13 plateaus of 16 measurements each for a total of 208 measurements. Furthermore, the frequency bandwidth of this measurement sequence is smaller than that of the Sheffield protocol. The measurement indexes increase from 1 to 416 in Fig. 9 since our system was set up to acquire each of the 208 measurements twice.

Close inspection of Fig. 9 shows that the shape of the SNR curve generally follows the shape of the average EIT signal. This clearly indicates than some of the noise sources have an additive contribution to the EIT signal. Some mea-

TABLE III

THEORETICAL ACCURACY EVALUATED ON PHANTOMS BUILT WITH RESISTORS WHOSE TOLERANCE IS $0.1 \%, 1 \%$, AND $5 \%$

\begin{tabular}{llccc}
\hline Tolerance (\%) & & 0.1 & 1 & 5 \\
\hline Accuracy (\%) & mean & 99.97 & 99.69 & 98.46 \\
& maximum & 100.0 & 100.0 & 100.0 \\
& minimum & 99.88 & 98.85 & 94.25 \\
\hline
\end{tabular}




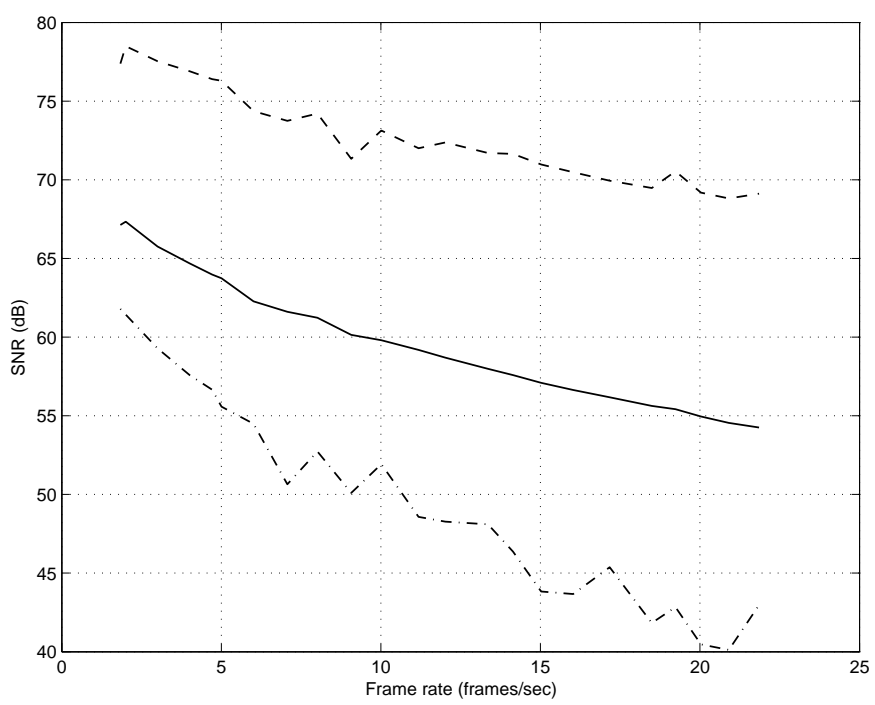

Fig. 11. SNR as a function of frame rate for our EIT system. (-·-) corresponds to the minimal SNR value, (-), the mean SNR value, and (---), the maxima SNR value.

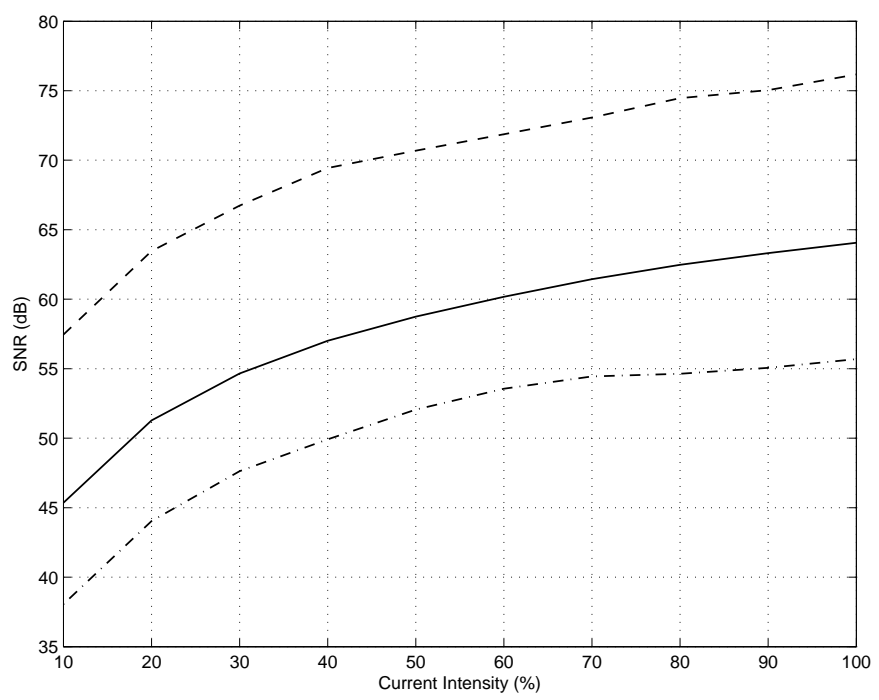

Fig. 12. SNR as a function of current intensity for our EIT system. (---) corresponds to the minimal SNR value, $(-)$, the mean SNR value, and (---), the maximal SNR value.

surement indexes, however, exhibit lower SNR values than their neighbors with similar signal amplitude. This corresponds in most cases to measurements that are performed using pairs of electrodes whose corresponding front-end electronic components are located at opposite ends of the EIT system PCB. By comparing the accuracy curve with the average EIT signal curve, the reader will notice that the accuracy is much lower whenever there is a large transition in the average EIT signal. This problem is related to a low-pass filter which is part of the demodulation process used in our system. Since this low-pass filter is part of the design, its impulse response is well known and has been integrated into the forward problem solver [12] to compute the modeling accuracy. This is why the modeling accuracy does not exhibit the large variations observed in the accuracy curve.

When evaluating accuracy and modeling accuracy with the proposed phantom, one limitation comes from the fact that the theoretical value for each measurement is calculated using the nominal values of the 340 resistors composing the phantom. In practice, the tolerance of the resistors that were used to build the phantom is $0.1 \%$. Part of the inaccuracy that is evaluated with the proposed method will therefore come from the uncertainty about the actual resistor values that were used to build the phantom. Therefore, the proposed method for evaluating the accuracy and modeling accuracy of an EIT system will, in some cases, underestimate the actual accuracy of the system due to the $0.1 \%$ tolerance of the resistors. In order to evaluate the contribution of the resistor tolerance to the inaccuracy of a system, we performed $2^{17}=131072$ Monte Carlo simulations where the resistor values of each simulated phantom were obtained using the upper and lower limits of the tolerance for each of the 17 different nominal resistor values. This assumption is based on the fact that resistors from the same production lot will generally exhibit an unknown but similar deviation from their nominal value within the specified limits of their tolerance. Table III summarizes the accuracy figures that were obtained for phantoms built from resistors whose tolerance is $0.1 \%, 1 \%$, and $5 \%$. Close inspection reveals that an EIT system whose accuracy has been evaluated at $99.88 \%$ using a phantom built with $0.1 \%$ precision resistors could actually have an accuracy of $100 \%$ since the $0.12 \%$ difference can theoretically be explained by the $0.1 \%$ tolerance of the resistors.

In order to validate the accuracy of our phantom by an independent method, we have measured the impedance of the 16 adjacent pairs of electrodes using a digital multimeter (34401A, Agilent Technologies). The 16 measured impedances varied from 409.17 to $409.42 \Omega$ with an average of $409.29 \Omega$. The theoretical value of the impedance computed using the resistor nominal values is $409.29 \Omega$. The maximum relative error on the measured impedance is therefore around $0.0318 \%$ which is, as expected, lower than the $0.1 \%$ tolerance of the resistors.

As shown by Gagnon [9] and Robitaille [14], performance indicators are affected by several parameters. For instance, by decreasing the operating frequency or the frame rate, performance indicators improve. They can be further improved by increasing the applied current intensity. To illustrate this fact, Fig. 11 shows how the SNR varies as a function of frame rate for our system while Fig. 12 shows how the SNR varies as a function of current intensity expressed as a percentage of the maximal current our system can apply which is 4 mApp. At 1.84 frames per second, the mean SNR is $67.13 \mathrm{~dB}$ while at 21.85 frames per second the mean SNR decreases to $54.25 \mathrm{~dB}$. At $100 \%$ applied current intensity, the mean SNR is $64.06 \mathrm{~dB}$ and at $10 \%$ current intensity the mean SNR decreases to $45.35 \mathrm{~dB}$. The accuracy and modeling accuracy were also computed from the same data set but remained constant at the values specified in Table II for all current intensities and frame rates. Although this is true for our EIT system, other EIT systems may behave otherwise. Measurement strategies also affect performance 


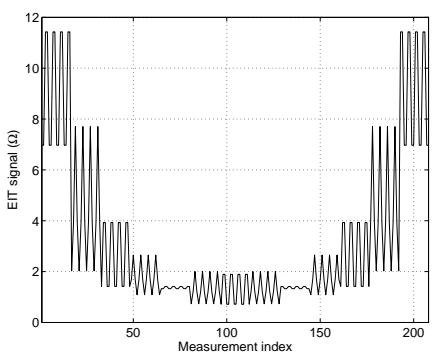

(a)

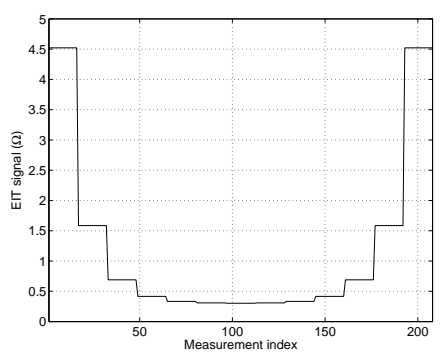

(c)

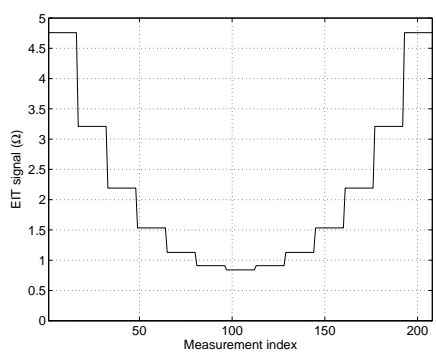

(b)

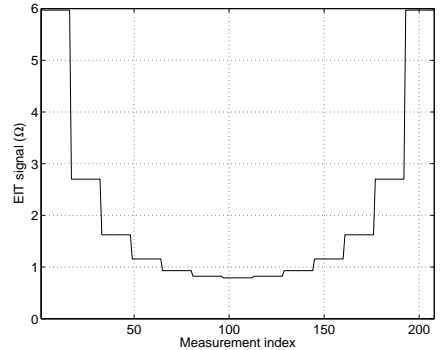

(d)
Fig. 13. a) Theoretical signal obtained from the Cardiff phantom. b) Theoretical signal obtained from the wheel phantom. c) Theoretical signal obtained from the first Göttingen phantom. d) Theoretical signal obtained from the proposed phantom. All theoretical signals were obtained using the measurement sequence described in Fig. 10.

indicators since they modify the amplitude dynamic range and frequency content of EIT signals. For multi-frequency systems, inter-modulation between the measurement frequencies may also adversely affect the performance indicators.

\section{DisCUSSION AND CONCLUSION}

Theoretical EIT signals produced by the Cardiff phantom [3], the wheel phantom [6], the first Göttingen phantom [4], and our phantom have been computed and are shown in Fig. 13. Since the resistor values for the wheel phantom are unspecified, their values were optimized to minimize the error between the signal it produces and the signal produced by our phantom. To obtain Fig. 13(b), resistors identified as (1) in Fig. 1(b) were therefore set to $56 \Omega$ while resistors identified as (2) were set to $330 \Omega$. Mainly due to the fact that the Cardiff phantom approximates a circular shape with square elements, the signal it produces [see Fig. 13(a)] does not exhibit the regular shape expected from a continuous circular conductive medium. The Cardiff phantom is, however, very good at producing localized conductivity perturbations since it contains 624 impedance elements [see Fig. 1(a)] that can be easily shunted. The first Göttingen phantom and the wheel phantom produce EIT signals with a shape similar to an actual EIT signal measured on a continuous circular homogeneous medium. They are however not very good at producing localized conductivity perturbations to test image reconstruction algorithms due to the fact that they are composed of few impedance elements especially in the middle [see Fig. 1(b) and (c)].

The phantom illustrated in Fig. 1(d) was recently proposed by the Göttingen group [5]. This phantom produces a constant signal for all measurements of the Sheffield protocol and its amplitude can be set by changing a single resistor value identified as (1) in Fig. 1(d). This phantom is great for exploring the strengths and weaknesses of an EIT system design and is well adapted to the design of the Göttingen EIT system. It cannot however be used to assess the performance of all EIT systems for the following reasons: 1) It does not support all measurement protocols such as those that use non-adjacent electrode pairs. 2) It generates a constant signal that does not reflect the frequency content or the amplitude dynamic range of a real EIT signal. It thus cannot be used to discriminate EIT systems that distort the EIT signal amplitude or its frequency content.

While performing in vivo data acquisitions, cables are used to connect the EIT system to the electrodes that are placed on the body. The assembly and layout of the cables introduce stray effects (mainly capacitive and inductive) which contribute noise to the EIT measurements. By using snap-on connectors, the same cables that are used for in vivo recording can be connected to our phantom as they would on a patient. By using cables to connect the Göttingen phantom to a DB37 connector and an adapter to connect the cables to the phantom, the cable assembly and layout is different from the one used for in vivo recording and, therefore, unrepresentative stray effects can be expected. Our phantom also incorporates a realistic electrical model of electrode impedances obtained from in vitro data while the Göttingen phantom has no equivalent. The set-up of our phantom is therefore more representative of cable and electrode stray effects that adversely affect EIT measurements during in vivo recording.

As our phantom is a resistor mesh with seventeen terminal nodes, one could argue that it could be simplified to use fewer resistor elements. While this is theoretically correct, the main purpose of this phantom is to assess the performance of EIT systems in a realistic predictable scenario. Although the phantom and its simplified version would produce the same EIT signals, contributions from error sources (stray effects, electromagnetic interference) would be different because they depend mainly on geometric factors. The simplified version would also lose the ability to produce localized conductivity perturbations which is very useful to compare reconstruction algorithms as was done in a previous study [12] using an early prototype version of the proposed phantom.

Since our phantom is composed entirely of resistors except for the capacitors that are part of the electrode equivalent model [see Fig. 7], its use to assess the performance of multifrequency EIT systems is limited by the fact that the conductivity distribution of the medium does not vary as a function of frequency. This is not however a limitation of the method since the proposed method is also applicable to complex impedance elements that could be defined as parallel or serial combinations of resistors, capacitors, and inductors, although inductive effects are very seldom seen in biological tissues.

Three performance indicators that can be computed from measurements made with this phantom have been presented. These performance indicators are function of the measurement index. It is therefore mandatory to represent them graphically 
or at least to specify minimum, mean, and maximum values rather than mentioning an ambiguous scalar value. Many factors influence the performance indicators of a system: measurement strategy, operating frequency, frame rate, applied current intensity, and inter-modulation distortion. Their values should therefore be specified with all performance indicators to better appreciate their significance.

The aim of this project was to build a phantom that produces realistic signals while taking into account stray effects similar to in vivo conditions. The phantom presented in this paper approximates a 2D circular continuous homogeneous medium. The amplitude dynamic range of the signals it produces is therefore limited by an order of magnitude compared to signals that would be obtained from a $3 \mathrm{D}$ conductive medium or in vivo. The ability of the current phantom to assess the performance of EIT systems in the small amplitude portion of EIT signals is therefore limited. A solution would be to modify the resistor values of the phantom to approximate a 3D circular homogeneous medium. The signals produced by the phantom would then have an increased amplitude dynamic range as expected from a $3 \mathrm{D}$ conductive medium. A further improvement would be to design a 3D torso-shaped phantom that would produce an even more realistic signal as far as frequency and amplitude range are concerned.

We are currently working on improving the capability of our phantom to assess the performance of EIT systems by adding programmable active components that can simulate contact impedance variations related to electrode movement artifacts. This improvement would be useful to test if the input impedance of the voltage amplifiers and the output impedance and compliance of the current sources are high enough to cope with the large contact impedance variations that are expected to occur during long term in vivo recording.

\section{REFERENCES}

[1] D. S. Holder, Electrical Impedance Tomography Methods, History and Applications. Bristol, U.K.: Inst. Phys. Pub., 2005.

[2] B. Rigaud and J. P. Morucci, "Bioelectrical impedance techniques in medicine. Part III: Impedance imaging. First section: general concepts and hardware," Crit. Rev. Biomed. Eng., vol. 24, no. 4-6, pp. 467-597, 1996.

[3] H. Griffiths, "A phantom for electrical impedance tomography," Clin. Phys. Physiol. Meas., vol. 9, suppl. A, pp. 15-20, 1988.

[4] G. Hahn, M. Beer, I. Frerichs, T. Dudykevych, T. Schröder, and G. Hellige, "A simple method to check the dynamic performance of electrical impedance tomography systems," Physiol. Meas., vol. 21, no. 1, pp. 5360, Feb. 2000.

[5] G. Hahn, A. Just, J. Dittmar, and G. Hellige, "Systematic errors of EIT systems determined by easily-scalable resistive phantoms," Physiol. Meas., vol. 29, no. 6, pp. S163-S172, June 2008.

[6] H. Griffiths, "A Cole phantom for EIT," Physiol. Meas., vol. 16, suppl. 3A, pp. A29-A38, Aug. 1995.

[7] I. D. Schneider, R. Kleffel, D. Jennings, and A. J. Courtenay, "Design of an electrical impedance tomography phantom using active elements," Med. Biol. Eng. Comput., vol. 38, no. 4, pp. 390-394, Jul. 2000.

[8] R. Kleffel, I. D. Schneider, and D. Jennings, "Synthesis of a digitally controlled impedance element," Med. Biol. Eng. Comput., vol. 38, no. 4, pp. 395-400, Jul. 2000.

[9] H. Gagnon, "Évaluation de la ventilation pulmonaire par tomographie d'impédance électrique," Ph.D. thesis, École Polytechnique de Montréal, Montréal, Canada, 2003.

[10] J. G. Webster, Medical Instrumentation Application and Design. NewYork: Wiley, 2009.

[11] D. W. Marquardt, "An algorithm for least-squares estimation of nonlinear parameters," SIAM J. Appl. Math, vol. 11, no 2, pp. 431-441, June 1963.

[12] A. E. Hartinger, H. Gagnon, and R. Guardo, "Accounting for hardware imperfections in EIT image reconstruction algorithms," Physiol. Meas., vol. 28, no. 7, pp. S13-S27, Jul. 2007.

[13] A. E. Hartinger, H. Gagnon, and R. Guardo, "A method for modelling and optimizing an electrical impedance tomography system," Physiol. Meas., vol. 27, no. 5, pp. S51-S64, May 2006.

[14] N. Robitaille, "Développement d'un système de tomographie d'impédance électrique multifréquence pour la détection de l'œdème pulmonaire," Ph.D. thesis, École Polytechnique de Montréal, Montréal, Canada, 2007.

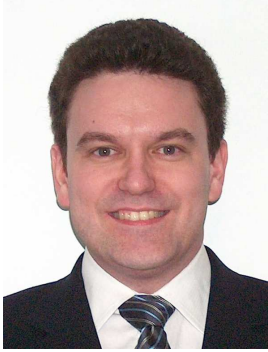

element methods.
Hervé Gagnon received the B.Sc.A. degree in electrical engineering from the Université Laval, Québec, QC, Canada, in 1994, and the M.Sc.A. and $\mathrm{Ph}$.D. degrees in biomedical engineering from the École Polytechnique de Montréal, Montréal, QC, in 1997 and 2003, respectively.

$\mathrm{He}$ is currently a Researcher at the École Polytechnique de Montréal. His current research interests include electrical impedance tomography, electronic design, real-time parallel processing, image reconstruction, optimization algorithms, and finite-

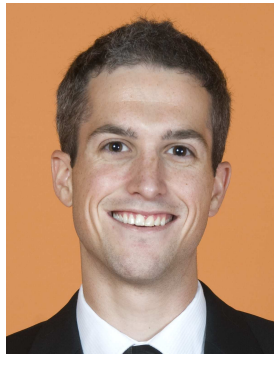

Martin Cousineau received the B.Ing. degree in mechanical engineering, biomedical engineering concentration, from the École Polytechnique de Montréal, Montréal, QC, Canada, in 2010. He is currently starting to work in the engineering field while pursuing the D.E.S.S. degree in management at HEC Montréal, Montréal, QC, Canada.

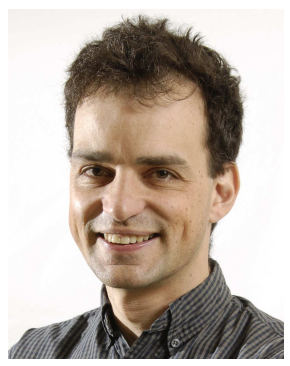

Andy Adler received the B.A.Sc. (honours) in engineering physics from University of British Columbia, Vancouver, Canada, in 1990, and a Ph.D. in biomedical engineering from École Polytechnique de Montréal, Montréal, Canada, in 1995.

$\mathrm{He}$ is currently an Associate Professor and Canada Research Chair (Tier II) in biomedical engineering at Carleton University, Ottawa, Canada. His research interests are in development of non-invasive biomedical measurement technologies, algorithms and sensors. He previously worked in senior technology positions at several companies.

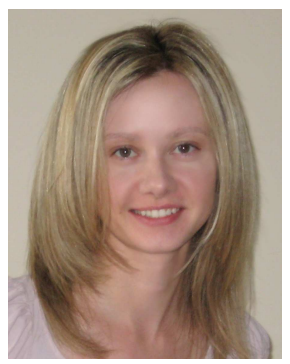

Alzbeta E. Hartinger (S'07) received the B.Ing. degree in electrical engineering in 2003 and the M.Sc.A. degree in biomedical engineering in 2006 from the École Polytechnique de Montréal, Montréal, QC, Canada, where she is currently working toward the $\mathrm{Ph} . \mathrm{D}$. degree in biomedical engineering.

Her current research interests include electrical impedance tomography, skin cancer detection, finiteelement methods, electronic design, and image reconstruction algorithms. 